\title{
Stock de carbone dans les gros débris ligneux végétaux : cas des forêts tropicales pluvieuses de la Likouala, République du Congo
}

\author{
Suspense Averti Ifo, Docteur \\ Mackline Mbemba, Etudiante
}

Ecole Normale Supérieure, Laboratoire de Géomatique et d'Ecologie

Tropicale Appliquée, Université Marien N'GOUABI, BP 69.

Brazzaville, République of the Congo

Félix Koubouana, Professeur

ENSAF, Laboratoire de Géomatique et d'Ecologie Tropicale Appliquée,

Université Marien N'GOUABI, BP 69,

Brazzaville, République of the Congo

Stoffenne Binsangou, Doctorante

Ecole Normale Supérieure, Laboratoire de Géomatique et d'Ecologie

Tropicale Appliquée, Université Marien N'GOUABI, BP 69.

Brazzaville, République of the Congo

doi: 10.19044/esj.2017.v13n12p384 URL:http://dx.doi.org/10.19044/esj.2017.v13n12p384

\begin{abstract}
In order to improve the knowledge of the functioning of tropical rainfall forests in the Congo basin ,a study on the quantification of the stocks of carbon in coarse woody debris was done in Likouala's area, in Republic of Congo. To achieve this goal,14 squares plots of $50 \mathrm{~m} \times 50 \mathrm{~m}$ had installed on the whole study area, including ten plots in primary forest and four in secondary forests. The method used was to make an inventory of woody debris lying on the ground or Log and snags in the different study plots. There sults of this study revealed average stocks of $603.45 \mathrm{~kg} \mathrm{MS} . \mathrm{ha}^{-1}$ in primary forests, $468.64 \mathrm{~kg} \mathrm{MS} . \mathrm{ha}^{-1}$ in secondaryforestsand392.68 kgMS.ha${ }^{1}$ in agroforestry, with no significant differences between stocks in primary forests and secondary forests $(\mathrm{P}=0.05)$. In addition this study revealed that carbon stocks varied from one locality to another in all the study area, as well as between the experimental plots $(\mathrm{p}=0.005)$. This study helps to understand that the Chablis played a key role in the production of large woody debris.
\end{abstract}

Keywords: Tropical Forest, Coarse Woody Debris, Log, AGB, Likouala 


\section{Résumé}

Dans le but d'améliorer la connaissance du fonctionnement des forêts tropicales pluvieuses dans le bassin du Congo, une étude sur la quantification des stocks de carbone dans les gros débris ligneux végétaux a été effectuée dans les forêts tropicales humides de la Likouala, en République du Congo. Pour atteindre cet objectif, 14 parcelles carrées de $50 \mathrm{~m}$ x $50 \mathrm{~m}$ ont été installées sur l'ensemble de la zone d'étude, dont dix parcelles dans les forêts primaires et quatre dans les forêts secondaires. La méthode utilisée a consisté à faire l'inventaire des débris ligneux végétaux couchés au sol ou $\log$ et des snags dans les différentes parcelles d'étude. Les résultats de cette étude ont révélé des stocks moyens de gros débris ligneux $603,45 \mathrm{~kg} \mathrm{MS} \mathrm{ha}^{-1}$ dans les forêts primaires, de 468,64 kg MS.ha ${ }^{-1}$ dans les forêts secondaires et de 392,68 kgMS.ha ${ }^{-1}$ en Agroforesterie, avec des différences non significatives entre les stocks des forêts primaires et les forêts secondaires $(\mathrm{P}=0,05)$. Par ailleurs cette étude a révélé que les stocks de carbone variaient d'une localité à une autre du sud vers le Nord, ainsi que entre les parcelles expérimentales $(\mathrm{p}=0,05)$. Cette étude a permis de comprendre que les chablis ont joué un rôle déterminant dans la production des gros débris ligneux végétaux.

Mots-clés : Forêt Tropicale, Débris Bois Mort, Log, AGB, Likouala

\section{Introduction}

Les débris ligneux végétaux constituent un des compartiments des écosystèmes forestiers, et ils interviennent dans la structure et dans le fonctionnement des écosystèmes forestiers (Ifo et al. 2015, Harmon et al., 1986 ; Samuelson et al. 1994 ; Francklin et al. 1987). Ils constituent un important réservoir de carbone dans les écosystèmes forestiers à la fois comme source et puits de $\mathrm{CO}_{2}$ pour l'atmosphère (Wendy et al. 2006, Ganjegunte et al., 2004 ; Baker et al., 2007). Les études de bois mort réalisées dans les forêts tempérées nord-américaines révèlent que, les gros débris ligneux constituent près de $12 \%$ de la masse totale de carbone de ces écosystèmes forestiers et que la quantité des débris ligneux végétaux varie considérablement d'un continent à un autre (Turner et al., 1995 ; Bhatti et al.,2002 in Gough et al., 2007).

Suivant le diamètre des débris ligneux végétaux, on distingue deux catégories: les gros débris ligneux et les petits débris ligneux (Wilcke et al., 2005 ; Woldendrop et al., 2004, Clark et al., 2002). Par ailleurs les scientifiques s'accordent sur l'existence de trois classes de gros débris ligneux (gdl) qui sont : gdl couchés au sol (en anglais 'Logs' (Pedlar et al. (2002)) ; Snags, qui sont des débris ligneux sur pieds et ayant une hauteur $\geq$ 1,5 m (Pedlar et al. 2002) ; Stump ou souche, pour le bois mort sur pieds et 
ayant une hauteur $\leq 1,5 \mathrm{~m}$. En ce qui concerne la définition des gdl, on note cependant que certains auteurs par contre incluent aussi les gdl non en contact direct avec le sol. Une fois cette catégorisation faite, à l'intérieur des 'logs' une classification est faite suivant le diamètre du bois mort, la longueur, etc. C'est ainsi qu'il y a des auteurs qui définissent les gros débris ligneux comme ayant un diamètre $>8-10 \mathrm{~cm}$ (Harmon et Sexton, 1996 ; Stevens, 1997 ; Idol et al., 2001 ; Stephens et Moghaddas, 2005; Wendy et al., 2006 ; Baker et al., 2007), alors que certains autres classifient ceux ayant un diamètre $<8-10 \mathrm{~cm}$ dans le groupe des petits débris ligneux (pdl) (Wendy et al., 2006). Aussi est-il important de faire remarquer que Barber et Van Lear (1984) considèrent les gdl comme les débris végétaux de diamètre $\geq 2,5$ $\mathrm{cm}$.

Très peu d'études d'inventaire de bois mort ont été réalisées dans les forêts du bassin du Congo, parmi les études existantes, on peut citer les travaux de Ifo (2010) dans deux types de forêts naturelles dans les Plateaux Batékés en République du Congo., mais aussi les travaux de Bocko et al. (2017) dans les forêts dans la Likouala. Ces derniers travaux ont été effectués dans une même zone d'étude que celle dans laquelle la présente étude a été effectuée.

La présente étude a lieu dans les forêts de la Likouala et plus spécifiquement dans les forêts de l'axe Impfondo Dongou caractérisé par 1'existence d'une superficie importante de forêt primaire à Lophira alata, des forêts Guibourtia demeusei, mais aussi une superficie non négligeable de forêt à Macaranga spp (Ifo et al. 2016).

L’inventaire spécifique du compartiment bois mort des forêts de la Likouala s'avère alors nécessaire pour estimer le stock total de carbone contenu dans ce compartiment. Aussi il est important de savoir que le compartiment bois mort est un indicateur de l'état d'équilibre d'un écosystème forestier. Ce qui, dans le contexte actuel devrait permettre d'accroitre les connaissances sur le fonctionnement des forêts du bassin du Congo en général et de celles de la Likouala en particulier ; et d'améliorer l'estimation sur le pourcentage de $\mathrm{CO}_{2}$ capté par ces forêts. Car, en effet les apports de carbone au sol dans les écosystèmes dépendent à la fois de la biomasse sur pied, des résidus d'arbres de toutes formes, de la durée de vie des organes (turnover), de la composition spécifique du peuplement, et de sa densité, des caractéristiques édaphiques et climatiques du site (Ifo, 2010).

L'objectif de la présente recherche est de faire une étude comparative des stocks de bois mort entre les forêts primaires (forêts intactes) et les forêts dégradées (forêts secondaires, agroforesterie) de la Likouala de l'axe Impfondo Dongou. 


\section{Méthodologie}

\subsection{Présentation de la zone d'étude}

Le zone d'étude se trouve dans la partie septentrionale de la république du Congo, plus précisément dans le département de la Likouala et couvre une superficie de 155274 ha. Ce département s'étend sur $230 \mathrm{Km}$ d'Est à l'Ouest et de $550 \mathrm{Km}$ environ du Nord au Sud, il est limitée : au Nord par la République Centrafricaine, au Sud par le département de la cuvette centrale, à l'Est par la République Démocratique du Congo, à l'Ouest par le Département de la Sangha (Figure 1).

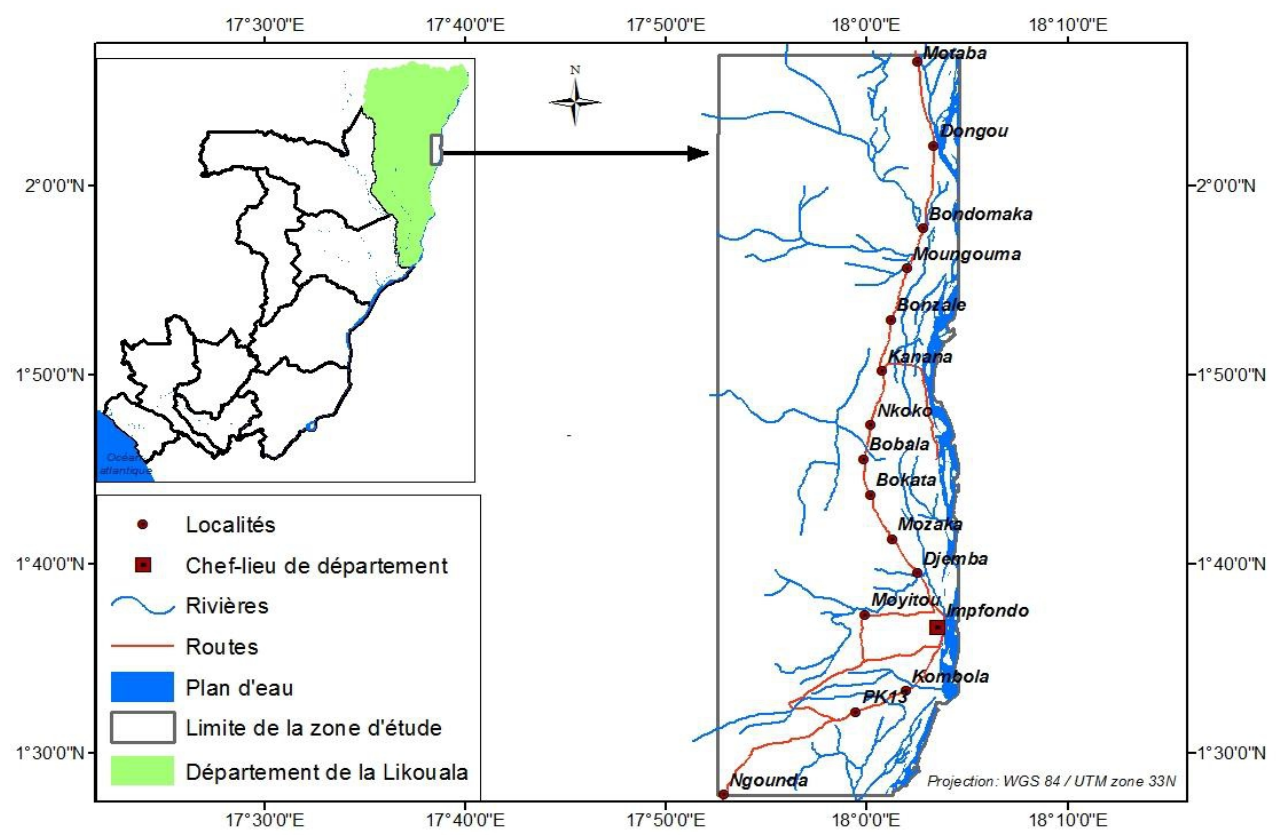

Figure 28 : Localisation de la zone d'étude

\subsection{Climat}

Le climat qui règne dans le département s'apparente aux climats équatoriaux et tropicaux humides du type guinéen forestier. La moyenne de la température dans le département de la Likouala est de $25{ }^{\circ} \mathrm{C}$ alors que la moyenne des précipitations est précipitations de 1600 à $1800 \mathrm{~mm}$ avec une variabilité interannuelle de 10 à $15 \%$ température moyenne annuelle de 25 $26^{\circ} \mathrm{C}$ avec une amplitude de 1 à $2{ }^{\circ} \mathrm{C}$ (Figure 2) 


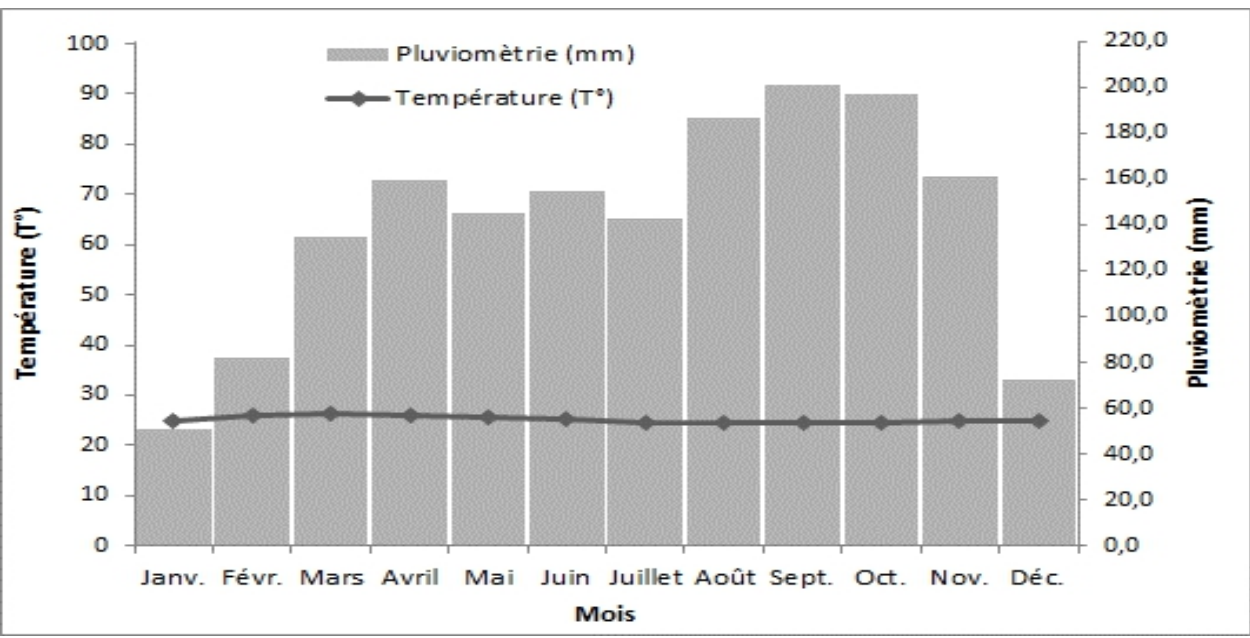

Figure 2 : Diagramme ombrothermique de la Likouala (moyenne de 1932-2015), ANAC Congo (2016)

\subsection{Végétation}

La végétation du département de la Likouala est essentiellement composée de forêts primaires et de forêts secondaires (figure 3). Cette carte d'occupation du sol a été faite à partir de la classification supervisée d'une image Landsat 8 grâce au logiciel de traitement d'images Envi 4.6. Le secteur forestier du Nord Congo, qui couvre les départements de la Likouala, la Sangha, la Cuvette et la Cuvette-Ouest, représente la plus vaste étendue de forêts du pays. C'est une forêt équatoriale qui couvre plus de 15 millions d'hectares dont plus de la moitié (9 millions d'hectares) de forêts continues sur sol ferme dominées par des essences de grande valeur commerciale telles le Sapelli (Entandrophragma cylindricum), le Sipo (Entandrophragma utile), le Wengué (Millettia laurentii) et le Padouk (Pterocarpus soyauxii). 


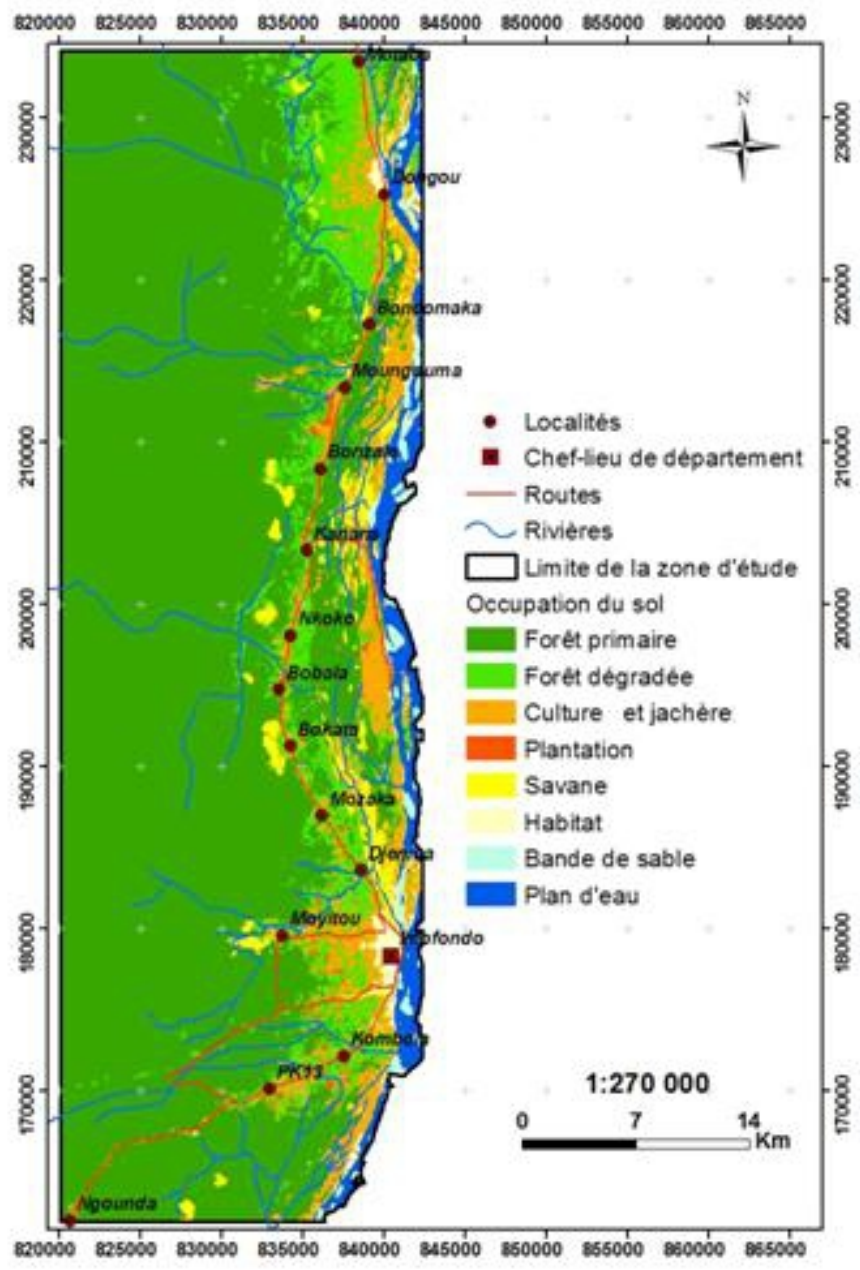

Figure 3 : carte Occupation des terres dans la zone d'étude

\subsection{Mise en place des parcelles expérimentales}

$\mathrm{Au}$ total 14 parcelles de forme carrée de $50 * 50 \mathrm{~m}$ chacune (soit $2500 \mathrm{~m}^{2}$ ou $0,25 \mathrm{ha}$ ), subdivisées en 56 placettes de $25 * 25 \mathrm{~m}$ ont été installées et inventoriées avec la répartition suivante: quatre parcelles de forêts dégradées ( 3 de forêts secondaires et une d'agroforesterie) et dix parcelles de forêts primaires (forêts intactes). La délimitation des parcelles a été réalisée à l'aide d'une boussole, suivant l'orientation Nord-Sud, Est-Ouest. Toutes les essences de DBH supérieur à $5 \mathrm{~cm}$ ont été inventoriées pour caractériser les différents types de forêt. Le tableau 1 présente le nombre d'individus par parcelle et suivant les types de forêt. 
Tableau 1 : Répartition des parcelles par type forestier

\begin{tabular}{|l|l|l|l|l|}
\hline Parcelles & Localités & type de forêts & Nombre de tiges & Biomasse aérienne (tC.ha-1) \\
\hline parcelle 07 & Bodzale & $\begin{array}{l}\text { forêt } \\
\text { indondée }\end{array}$ & 55 & 132,1 \\
\hline parcelle 08 & Bodzale & $\begin{array}{l}\text { forêt } \\
\text { indondée }\end{array}$ & 61 & 95,5 \\
\hline parcelle 10 & Bodzale & terre ferme & 106 & 87,4 \\
\hline Parcelle 11 & Bodzale & terre ferme & 217 & 73,9 \\
\hline parcelle 9 & Bodzale & terre ferme & 34 & 61,5 \\
\hline parcelle 1 & dongou & terre ferme & 67 & 18,9 \\
\hline parcelle 2 & dongou & terre ferme & 79 & 20,4 \\
\hline parcelle 3 & dongou & terre ferme & 212 & 101,2 \\
\hline parcelle 4 & dongou & terre ferme & 132 & 98,4 \\
\hline parcelle 5 & dongou & terre ferme & 111 & 80,2 \\
\hline parcelle 6 & dongou & $\begin{array}{l}\text { forêt } \\
\text { indondée }\end{array}$ & 102 & 96,5 \\
\hline parcelle 12 & impfondo & semi inondée & 126 & 63,9 \\
\hline parcelle 13 & impfondo & semi inondée & 153 & 109,3 \\
\hline parcelle 14 & impfondo & semi inondée & 109 & 68,6 \\
\hline parcelle 15 & impfondo & terre ferme & 47 & 108,9 \\
\hline
\end{tabular}

$\mathrm{FS}=$ forêt secondaire, $\mathrm{FP}=$ forêt primaire

Tableau 2 : Répartition des groupements forestiers suivant les parcelles d'étude

\begin{tabular}{|l|l|}
\hline parcelle 07 & Forêt à Guibourtia demeusei(Harms) Léon \\
\hline parcelle 08 & Forêt à Musanga cecropioides R. Br.ex Tedlie \\
\hline parcelle 1 & Forêt à Macaranga spinosa Müll.Arg. \\
\hline parcelle 10 & Forêt à Celtis adolphi-friderici Engl \\
\hline Parcelle 11 & Forêt à Musanga cecropioides R. Br.ex Tedlie \\
\hline parcelle 12 & Forêt à Lophira alata Banks ex Gaertu \\
\hline parcelle 13 & Forêt à Lophira alata Banks ex Gaertu \\
\hline parcelle 14 & Forêt à Lophira alata Banks ex Gaertu \\
\hline parcelle 15 & Forêt à Celtis adolphi-friderici Engl \\
\hline parcelle 2 & Forêt à Macaranga spinosa Müll.Arg. \\
\hline parcelle 3 & Forêt à Macaranga spinosa Müll.Arg. \\
\hline parcelle 4 & Forêt à Lophira alata Banks ex Gaertu \\
\hline parcelle 5 & Forêt à Guibourtia demeusei(Harms) Léon \\
\hline parcelle 6 & Forêt à Guibourtia demeusei(Harms) Léon \\
\hline parcelle 9 & Forêt à Guibourtia demeusei(Harms) Léon \\
\hline
\end{tabular}

Nous avons défini les gdl comme étant tous les débris ligneux végétaux couchés en contact direct ou non avec le sol et ayant un diamètre $\geq$ $2,5 \mathrm{~cm}$. A l'intérieur des parcelles installées, les échantillons de bois mort ont été collectés en tenant compte des trois catégories suivantes :les gdl (gros 
débris ligneux) ou bois morts couchés au sol en anglais 'Logs' (Pedlar et al. 2002), les gdl sont définis dans cette étude comme étant tous les débris ligneux couchés, en contact direct ou non avec le sol et ayant un diamètre $>$ $2,5 \mathrm{~cm}$, les 'Snags', ce sont des débris ligneux sur pieds d'une hauteur $\geq 1,5$ m (Pedlar et al. 2002);

\section{Collecte des logs}

Pour collecter les logs nous avions procédé par un inventaire spécifique de tous les débris ligneux couchés, en contact direct ou non (allongé en prenant appui sur arbre vivant ou sur un autre bois mort) avec le sol et ayant un diamètre supérieur ou égale à $2,5 \mathrm{~cm}$.

\section{Collecte des Snags}

La collecte des Snags a consisté en un inventaire de tous les échantillons de bois mort sur pied d'une hauteur supérieure ou égale à $1,5 \mathrm{~m}$. La mesure du diamètre et de la hauteur est faite.

Le diamètre de chaque échantillon de bois mort est mesuré à $1,30 \mathrm{~m}$ du sol ou à $30 \mathrm{~cm}$ au-dessus des contreforts (pour les arbres morts à contreforts) à l'aide d'un ruban métrique.

Pour les échantillons d'une hauteur inférieure ou égale à $2 \mathrm{~m}$, la hauteur est mesurée à l'aide du ruban métrique. Pour ceux d'une hauteur supérieure à 2 $\mathrm{m}$, la hauteur est mesurée à l'aide d'un dendromètre de marque Suuto.

\subsection{Méthode de calcul des volumes}

\subsection{Calcul du volume des Logs}

Pour calculer le volume des logs nous avons utilisé l'équation 2.1 proposée par Waren et Olsen (1964) :

$$
v=\frac{\pi^{2}\left(\sum d i^{2}\right)}{8 L}
$$

Equation 2.

$\mathrm{V}=$ volume de gros débris ligneux $\left(\mathrm{m}^{3} \cdot \mathrm{ha}^{-1}\right)$, di, est le diamètre de chaque débris ligneux échantillonné $(\mathrm{m}), \mathrm{L}=100$. La conversion des résultats obtenus du volume en masse est obtenue en fixant la valeur de la densité du bois à $0,5 \mathrm{KgMS} . \mathrm{m}^{-3}$.

\subsubsection{Calcul du volume des snags}

Le volume des snags a été calculé, à partir de l'équation 3

$$
V s=\left(\frac{d b h}{2}\right)^{2} \times \pi \times h \times f
$$


VS= Volume snag $(\mathrm{m} 3) ; \mathrm{dbh}=$ diamètre à la hauteur du sein $(\mathrm{m}) ; \mathrm{h}=$ hauteur du snag $(m)$,

$\mathrm{f}=$ facteur forme $(0,627)$. La conversion en masse a également été faite en fixant la valeur de la densité du bois à $0,5 \mathrm{KgMS} . \mathrm{m}^{-3}$.

\subsection{Analyse statistique}

L'analyse des données s'est faite grâce aux tableurs Excel. Sur ces tableurs, nous avons procédé à la mise en forme de la totalité des données brutes collectées sur le terrain. Cette mise en forme a consisté premièrement à l'épurement et l'harmonisation de toutes les données ce qui a permis d'aboutir à une synchronisation des différentes informations inscrites (localité, parcelle, numéro échantillon, nom local, nom scientifique...). Le volume, la masse sèche et la masse de carbone de chaque échantillon ont ensuite été calculés, le volume grâce au calcul à l'aide des équations en fonction des catégories de bois mort et les masses par conversion. Les graphiques ont été faits grâce au tableur Excel 2013.

\section{Résultats}

Variabilité spatiale du stock de carbone contenu dans logs entre les sites d'étude

Les stocks de carbone dans les logs différent entre les trois types de forêts : forêt primaire, forêt secondaire et l'agroforesterie. Le stock le plus faible a été obtenu dans les forêts de Bondzale avec 1129,53Kg.C.ha ${ }^{-1}$, alors que le stock le plus important a été enregistré dans les forêts de Dongou avec 1325,55 Kg.C.ha ${ }^{-1}$ (Figure 4). Le test statistique révèle une différence significative entre ces trois sites $(\mathrm{p}=0,05)$.

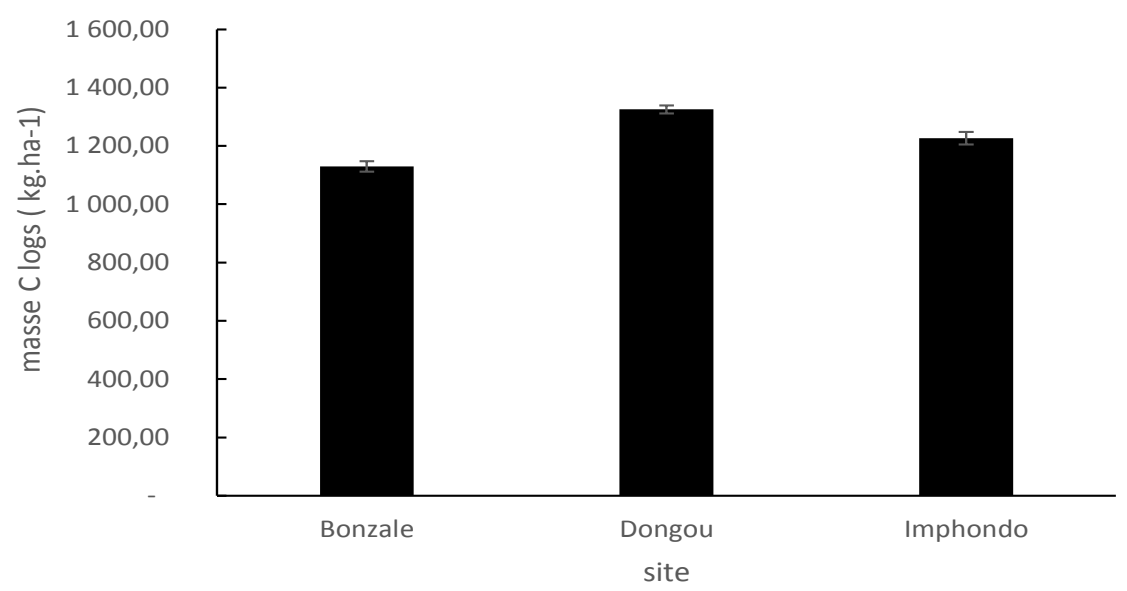

Figure 4: variabilité spatiale entre les trois sites d'étude du stock de carbone contenu dans les logs 
Variabilité spatiale du stock de carbone dans les logs entre les parcelles expérimentales

L'étude de la variabilité spatiale du stock de carbone contenu dans les logs entre les parcelles expérimentales révèle une grande variabilité entre les parcelles d'étude. Ce stock varie de $30 \mathrm{Kg}$.C.ha ${ }^{-1}$ à $380 \mathrm{Kg} . C . h a^{-1}$ de ce résultat nous avons noté qu'il y a des parcelles avec un stock de carbone inferieur à 100 kg.C.ha ${ }^{-1}$ (parcelles 1 et 7), mais aussi des parcelles ayant un stock de carbone supérieur à $350 \mathrm{Kg} \cdot \mathrm{C} \cdot \mathrm{ha}^{-1}$ (parcelles $12,13,3$ et 9). La parcelle 11 qui contient un peuplement pur à Musanga cecropioides contient un stock important de débris ligneux végétaux supérieur à $300 \mathrm{Kg}$.C.ha ${ }^{-1}$. (figure 5).

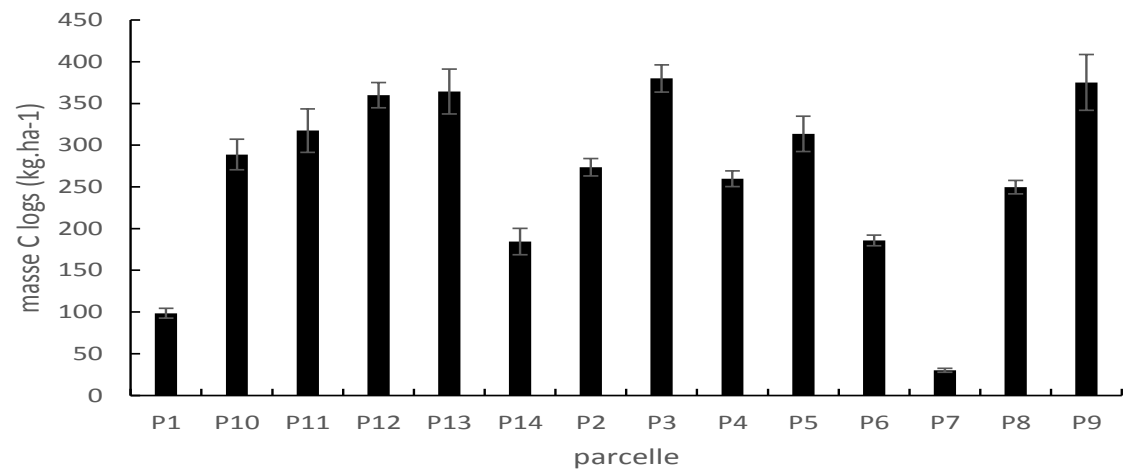

Figure 5: variabilité spatiale du stock de carbone contenu dans les logs entre les parcelles expérimentales

\section{Stock de carbone dans les Snags entre les trois localités d'étude.}

Pour les snags, la variabilité spatiale pour les snags varie entre les types de forêts. Dans un ordre décroissant les stocks de carbone étaient de 43,46 Kg.C.ha ${ }^{-1}$ à Dongou, de 16,69 Kg.C.ha ${ }^{-1}$ à Bondzale et de 2,45 à Impfondo. Le test de comparaison multiple indique que les stocks sont significativement différents.

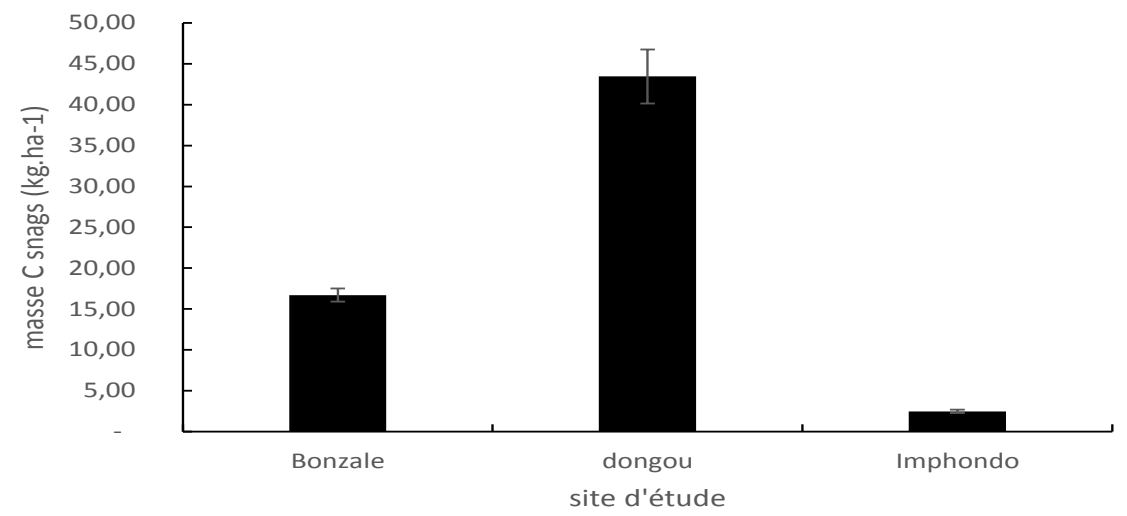

Figure 6: Variabilité spatiale du stock de carbone contenu dans les snags entre les trois 


\section{Stock carbone des Snags entre les parcelles expérimentales}

Le stock en masse de carbone contenu dans les snags entre les 14 parcelles d'étude varie considérablement de $0,02 \mathrm{Kg} \mathrm{C} \cdot \mathrm{ha}^{-1}$ (parcelle 8 ) à 16,10 Kg.C.ha ${ }^{-1}$ (parcelle 3).Sur l'ensemble des 14 parcelles, seulement 4 parcelles ont un stock de carbone au-dessus de $5 \mathrm{KgC}_{\mathrm{hah}}{ }^{-1}$.

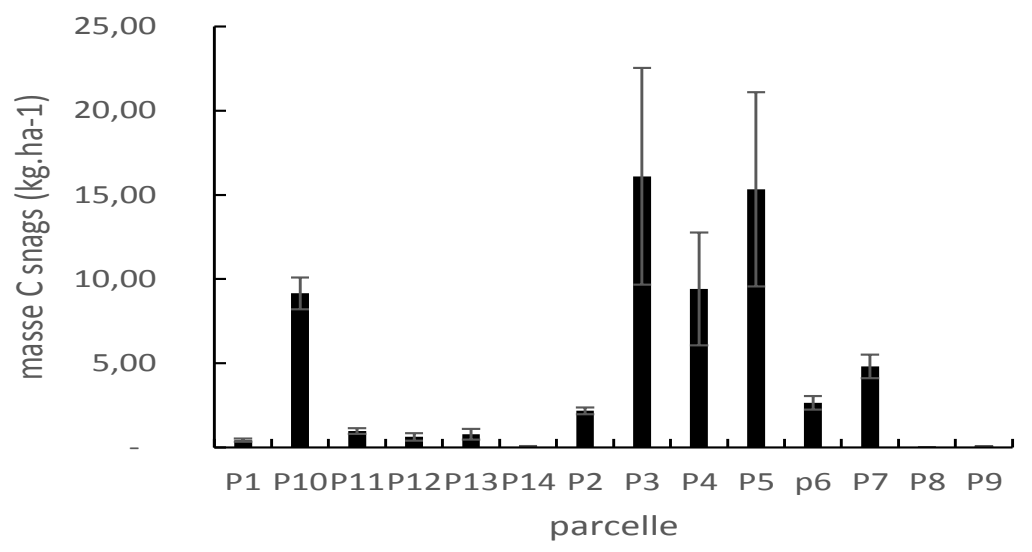

Figure 07: Variabilité spatiale du stock de carbone contenu dans les snags entre les parcelles expérimentales

Tableau 3: liste des cinq espèces les plus répandues dans les trois types de formation végétale

\begin{tabular}{|c|c|c|c|}
\hline $\begin{array}{ll}\mathrm{N}^{\circ} & \mathrm{de} \\
\text { l'espèce } & \end{array}$ & F P & F S & Ag \\
\hline 1 & Uapaca guineensis & $\begin{array}{l}\text { Musanga cecropioides } \\
\text { R. Br.ex Tedlie }\end{array}$ & $\begin{array}{l}\text { Strambosia } \\
\text { grandifolia }\end{array}$ \\
\hline 2 & Dialum pachyphyllum & $\begin{array}{l}\text { Petersianthus } \\
\text { macrocarpus }\end{array}$ & $\begin{array}{l}\text { Garcinia punctata } \\
\text { Oliv. }\end{array}$ \\
\hline 3 & Penthacletra macrophylla & $\begin{array}{l}\text { Strambosia } \\
\text { grandifolia }\end{array}$ & $\begin{array}{l}\text { Musanga } \\
\text { cecropioides R. Br.ex } \\
\text { Tedlie }\end{array}$ \\
\hline 4 & $\begin{array}{l}\text { Angylocalyx pynaertii De } \\
\text { Wild }\end{array}$ & $\begin{array}{l}\text { Penthacletra } \\
\text { macrophylla }\end{array}$ & $\begin{array}{l}\text { Angylocalyx pynaertii } \\
\text { De Wild }\end{array}$ \\
\hline 5 & Albizia laurentii & Uapaca guineensis & Panda oleosa \\
\hline
\end{tabular}

\section{Discussion}

Les stocks de débris ligneux végétaux obtenus lors de notre étude montrent une grande variabilité spatiale entre les types de forêts dans la zone d'étude qui couvre plusieurs types de forêt : forêt primaire, forêt dégradée et 
agroforesterie. Aussi il est important de dire dans nous avons identifié plusieurs groupements forestiers à partir de l'inventaire qui a été fait dans les différentes parcelles d'étude.

Suivant la catégorie de débris ligneux végétaux : Logs ou Snags nous avons noté une grande variation du stock gdl. Les stocks obtenus lors de cette étude sont plus importants par rapport aux résultats obtenus par d'autres auteurs dans d'autres types de forêt. (Baker et al. 2007 ; Grove et al. 2005).Plusieurs facteurs pourraient expliquer les variations de stocks des débris ligneux végétaux dans la zone d'étude : le type de forêt, l'âge, la structure et la dynamique des forêts étudiées (Siipola et al. 1998). En dehors de ces facteurs, nous pouvons aussi citer les phénomènes météorologiques (ouragans, tempêtes), mais aussi les phénomènes naturels de chablis. L'action des paramètres météorologiques a été notée par plusieurs autres auteurs parmi lesquels Harmon et al. (1986). Ils affirment que parmi les facteurs qui contribuent à la production des débris ligneux végétaux mais aussi à leur accumulation sur les sols forestiers, il y a l'action des tempêtes.

Du type de forêt, les stocks élevés notés dans les forêts primaires sont essentiellement dus aux phénomènes des chablis. Les bois morts enregistrés dans ces parcelles avaient des gros diamètres en comparaison des gros débris ligneux enregistrés dans les autres parcelles dans lesquelles l'inventaire avait été fait. A l'endroit où ces bois ont été enregistrés, la hauteur des arbres était moyenne en comparaison de des grands arbres tout autour. Par ailleurs cette hypothèse pourrait se justifier par le fait que le système racinaire de ces arbres était totalement exposé suite au renversement de l'arbre lors de sa chute. Au contraire des forêts primaires, les forêts secondaires ont un stock faible par le fait que les forêts sont en pleine croissance, ont des essences ayant encore des petits diamètres avec une hauteur moyenne moins importante que les forêts primaires qui sont des forêts matures, avec des gros diamètres et des arbres ayant une hauteur supérieure à 40 mètres et plus exposés à l'action d'autres facteurs tels que les vents. Aussi il est important de noter qu'aucun groupement forestier n'a été épargné par ce phénomène de chablis dans les forêts primaires. Cette étude a permis par ailleurs de découvrir que dans ces forêts primaires, la quantité enlevée de gros débris ligneux pourrait être un indicateur important du stade de développement d'un écosystème forestier.

Les forêts secondaires dans cette étude ont produit moins de stock que les forêts primaires. Dans les forêts secondaires et en agroforesterie, le ramassage des résidus d'arbres après coupe par les Hommes pourrait justifier la faible accumulation des débris ligneux dans ces deux systèmes forestiers. Par ailleurs les résultats de cette étude permettent de comprendre que les forêts primaires continuent à émettre des quantités non négligeables de $\mathrm{CO}_{2}$ dans l'atmosphère suite à la respiration hétérotrophe provenant de la 
décomposition des débris ligneux végétaux. Le tableau 3 révèle que beaucoup d'essences forestières contribuent au bois mort dans les différents types forestiers. Cette diversité végétale pourrait constituer un apport nutritionnel important pour l'écosystème à travers la libération des bios éléments lors de la décomposition de ces débris ligneux végétaux.

\section{Conclusion}

La présente étude basée sur l'inventaire de bois mort dans les forêts tropicales pluvieuses du Nord-Congo a permis de faire un point des connaissances d'une part sur le compartiment bois mort et de l'autre sur le rôle que joue ce compartiment dans le fonctionnement général des forêts tropicales pluvieuses en terme de quantification du carbone. Cette étude a montré que les forêts primaires avaient des stocks de bois mort plus important que ceux enregistrés dans les forêts secondaires et dans le système agroforesterie.

Les facteurs endogènes (vieillissement de l'écosystème forestier) ou des facteurs externes comme l'action des vents très forts pourraient expliquer les différences observées dans les trois types de forêts de la zone d'étude. Cette étude a montré par ailleurs que le compartiment bois mort doit être pris en compte dans la quantification des stocks de carbone des écosystèmes terrestres tropicaux.

Les résultats obtenus lors de cette étude restent à améliorer en augmentant la superficie des parcelles expérimentales dans la zone d'étude. Nous envisageons aussi faire une étude sur les classes de décomposition des gros débris ligneux ainsi que les espèces qui participent le plus à la décomposition des débris ligneux dans cette zone d'étude.

\section{References:}

1. Baker T.R., Coronado E.N.H., Phillips O.L., Martin J., Van der Heijden G.M.F., Garcia M. et Espejo J.S., 2007. Low stocks of coarse woody debris in a southwest Amazonian forest. Oecologia 152: 495504.

2. Barber B.L. et Van Lear D.H., 1984. Weight loss and nutrient dynamics in decomposing woody loblolly pine slash. Soil Science of America Journal 48: 906-910.

3. Bocko Y. E., Ifo S. A. Loumeto J. J. (2017). Quantification Des Stocks De Carbone De Trois Pools Clés De Carbone En Afrique Centrale : Cas De La Forêt Marécageuse De La Likouala (Nord Congo). European Scientific Journal February 2017 edition vol.13, No.5 ISSN: 1857 - 7881 (Print) e - ISSN 1857- 7431

4. Clark D.B., Clarck D.A., Brown S., Oberbaver S.F. et Veldkamp E., 2002. Stocks and flows of coarse woody debris across a tropical rain 
forest nutrient and topography gradient. Forest Ecology and Management. 164: 237-248.

5. Franklin J.F., Shugart H.H. et Harmon M.E., 1987. Tree death as an ecological process. The causes, consequences, and variability of tree mortality. Bioscience 37: 550-556.

6. Ganjegunte G.K., Condron L.M., Clinton W.P, Davis M.R. et Mahieu N., 2004 Decomposition and nutrient release from radiata pine (Pinus radiata) coarse woody debris. Forest Ecology and Management. 187: 197-211.

7. Génot P. et Génot J.C., 2005. Le bois mort est bien vivant. Syco parcdossier. www. Parc-Vosges. Fr.

8. Gough C.M., Vogel C.S., Kazanski C., Nagel L.N., Flower C.E. et Curtis P.S., 2007. Coarse woody debris and the carbon balance of a north temperate forest. Forest Ecology and Management 244: 60-67.

9. Goodburn J.M. and Lorimer C.G., 1998. Cavity trees and coarse woody debris in old-growth and managed northern hardwood forests in Wisconsin and Michigan. Canadian Journal of Forest Research, 28: 427-438.

10. Grove, J.S. (2005). Extent and Composition of dead wood in Australian lowlandtropical rainforest with different management histories. Forest Ecology andManagement 154: 35-53.

11. Harmon M.E., Franklin J.F., Swanson F.J., Sollins P., Gregory S.V., Lattin J.D., Anderson N.H., Cline P., Aumen N.G., Sedell J.R., Lienkaemper G.W., Cromack J.K. et Cummins K.W., 1986. Ecology of coarse woody debris in temperate ecosystems. Adv. Ecol. Res. 15: 133-302.

12. Harmon M.E. et Sexton J., 1996. Guidelines for Measurements of Woody Detritus in Forest Ecosystems (US LTER Publication No. 20) US LTER Network Office, University of Washington, Seattle. WA, USA. 73 pp.

13. Idol T.W., Figler R.A., Pope P.E. et Ponder Jr. F., 2001. Characterization of coarse woody debris accross a 100 year chronosequence of upland oak-hickory forests. Forest ecology and management. 149: 153-161.

14. Ifo A.S., Koubouana F., Jourdain C., et Nganga D. (2015). Stock and Flow of Carbon in Plant Woody Debris in Two Different Types of Natural Forests in Bateke Plateau, Central Africa. Open Journal of Forestry, 5, 38-47.

15. Ifo S. A, Moutsambote J.M, Koubouana F, Yoka J, Ndzai S.F, Bouetou-Kadilamio L.N. O, Mampouya H, Jourdain C, Bocko Y, Mantota A. B, Mbemba M, Mouanga-Sokath D, Odende R, Mondzali R. L, Mampouya Wenina Y. E, Ouissika B. C, and Loumeto J. J. 
(2016). Tree Species Diversity, Richness, and Similarity in Intact and Degraded Forest in the Tropical Rainforest of the Congo Basin: Case of the Forest of Likouala in the Republic of Congo. International Journal of Forestry Research Volume 2016, 12 pages. http://dx.doi.org/10.1155/2016/7593681

16. Pedlar J.H., Pearce J.L., Venier L.A. et McKenney D.W., 2002. Coarse woody debris in relation to disturbance and forest type in boreal Canada. Forest Ecology and Management 158: 189-194.

17. Pichery Ch., 2001. Eléments de réflexion pour une meilleure gestion du bois mort en forêt. Rapport ENGREF/FIF, Nancy, 95 pages + annexes.

18. Pittman R.J., 2005. Coarse woody debris in industrially managed Pinus taeda plantation of Southestern United States, MSc. Thesis, Forestry, Polytehnics Institute and State University, Virginia. 113 pages.

19. Samuelsson, J., Gustafsson, L., Ingelo, G,T., 1994. Dying and dead trees-areview of their importance for biodiversity. ArtDatabanken, Uppsala

20. Siipola A.L., Siitonen J. et Kallio R., 1998. Amount and quality of coarse woody debris in natural and managed coniferous forest near the timberline in Finnish Lapland. Scand. Jour. For. Res. 13: 204214.

21. Stephens S.L., Fry D.L, Franco-Vizcaino E., Collins B.M. et Moghaddas, J.M., 2007. Coarse woody debris and canopy cover in an old-growth Jeffrey pine-mixed conifer forest from the Sierra San Pedro Martir, Mexico. Forest Ecology and Management. 240: 87-95.

22. Stevens V., 1997. The ecological role of coarse woody debris: an overview of the ecological importance of CWD in B.C. forests. B.C. Ministry of Forests, Research Branch. Victoria, B.C. Work Pap. 30.

23. Turner D.P., Koerper G.J., Harmon M.E. et Lee J.J., 1995. A carbon budget for forests of the conterminous United States. Ecol. Appl. 5: 421-436.

24. Warren, W.G. and Olsen, P.F. (1964). A line intersect technique for assessinglogging waste. Forest Science 10: 267-276.

25. Wendy H.L., David M.B., Lucy R.H., Scott R.S., Elizabeth H.P., Daniel C. et Steven C. W., 2006. Woody debris contribution to the carbon budget of electively logged and maturing mid-latitude forests. Oecologia 148: 108-117.

26. Wilcke W., Hess T., Bengel C., Homeier J. et Valarezod C., 2005. For Ecology and

27. Management. Forest Ecology and Management. 75: 135-146. 
28. Woldendorp G., Keenan R.J., Barry S. et Spencer R.D., 2004. Analysis of sampling methods for coarse woody debris. Forest Ecology and Management. 198: 133-148.

29. Wilcke, W., Hess, T., Bengel, C., Homeier, J., Valarezod, C. 2005. For Ecology andManagement. Forest Ecology and Management. 75: 135-146.

30. Woldendorp, G., Keenan, R.J., Ryan, M.F. (2002). An Analysis of samplingMethods for Coarse Woody Debris in Australian Forest Ecosystems A Report for theNational Greenhouse Strategy, Module 6.6 (Criteria and Indicators of SustainableForest Management). 94 p. 